\title{
Análisis del ranking Great Place to Work y de los premios del Observatorio de Comunicación Interna: prácticas de comunicación interna en empresas españolas (2014-2018)
}

\author{
Analysis of the Great Place to Work and the Internal \\ Communication Observatory awards: Internal \\ communication practices in Spanish \\ companies (2014-2018)
}

\author{
Andrea Castro-Martínez; Pablo Díaz-Morilla
}

Cómo citar este artículo:

Castro-Martínez, Andrea; Díaz-Morilla, Pablo (2019). “Análisis del ranking Great Place to Work y de los premios del Observatorio de Comunicación Interna: prácticas de comunicación interna en empresas españolas (2014-2018)". El profesional de la información, v. 28, n. 5, e280518.

https://doi.org/10.3145/epi.2019.sep.18

Artículo recibido el 10-04-2019 Aceptación definitiva: 17-06-2019

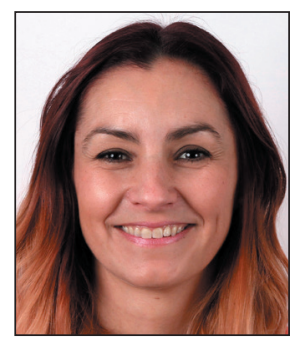

Andrea Castro-Martínez $\square$ https://orcid.org/0000-0002-2775-625X

Universidad de Málaga

Facultad de Ciencias de la Comunicación León Tolstoi, s/n. 29010 Málaga, España andreacastro@uma.es

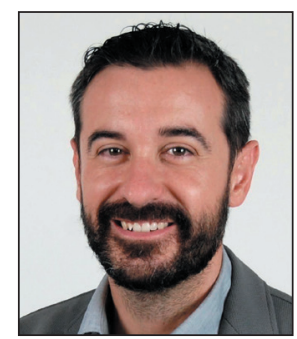

Pablo Díaz-Morilla

https://orcid.org/0000-0002-7914-3391

Universidad de Málaga y EADE Málaga Facultad de Ciencias de la Comunicación León Tolstoi, s/n. 29010 Málaga, España pablodmorilla@uma.es

\section{Resumen}

La comunicación interna es un valor en alza en el entorno empresarial debido a su utilidad para aumentar el compromiso de los empleados hacia su organización. Por ello constituye un campo de interés tanto para investigadores como para el tejido empresarial, que puede rentabilizar los avances. El objetivo de este trabajo es analizar las estrategias de las empresas españolas que son reconocidas por sus buenas prácticas en comunicación interna. Este estudio realiza un análisis secundario de datos obtenidos del ranking Great Place to Work y de los premios del Observatorio de Comunicación Interna e Identidad Corporativa entre 2014 y 2018, así como entrevistas semiestructuradas a representantes de ambas entidades. Los resultados muestran la consolidación de medios como embajadores de marca y aplicaciones internas, así como la tendencia a fomentar la experiencia de los empleados para aumentar su implicación y facilitar la gestión del cambio.

\section{Palabras clave}

Comunicación interna; Comunicación organizacional; Comunicación corporativa; Felicidad laboral; Compromiso organizacional; Herramientas internas; Relaciones públicas; Marketing interno; Organizaciones; Empresas; Intranets; Rankings; GPTW; Premios; OCl.

\section{Abstract}

Internal communication is a rising value in the business environment due to its usefulness to achieve the commitment of employees towards the organization. Therefore, it is an area of interest for both researchers and the business sector, which can make profitable advances. The objective of this paper is to analyze the strategies of Spanish companies that are recognized for their good practices in internal communication. This study performs a secondary analysis of the data 
Great Place to Work ranking and the Observatory of International Communication and Corporate Identity awards between 2014 and 2018, as well as semi-structured interviews with representatives of both entities. The results show the consolidation of tools as brand ambassadors and internal applications, as well as the trend to promote the experience of employees to increase their engagement and make change management possible.

\section{Keywords}

Internal communication; Organizational communication; Corporate communication; Work happiness; Organizational commitment; Internal tools; Public relations; Internal marketing; Organizations; Companies; Intranets; Rankings; GPTW; Awards; $\mathrm{OCl}$.

\section{Introducción}

En el actual entorno altamente competitivo, cambiante y saturado de mensajes publicitarios, la comunicación interna se ha convertido en una de las áreas de máxima importancia en la estructura organizacional. A través de la comunicación hacia su interior muchas empresas han fortalecido los procesos de identificación de los empleados hacia la marca, y han logrado amplificar el mensaje corporativo de forma que alcance a sus públicos internos y se expanda a través de ellos hacia el exterior de la organización (Malhotra; Ackfeldt, 2016).

Los instrumentos internos de comunicación se han modernizado y han incorporado a las ventajas de los antiguos métodos otras propuestas más novedosas para optimizar el alcance a los públicos internos. Este proceso de mejora e incremento de la comunicación interna es paralelo al creciente interés sobre la felicidad laboral. Por ello la felicidad en el trabajo ha ganado importancia en los últimos años y está siendo estudiada desde diferentes perspectivas (Fisher, 2010; Salas-Vallina; Alegre; Fernández-Guerrero, 2018), como la comunicación organizacional (Cheney et al., 2008; Proctor, 2014).

Este trabajo se centra en el modo en que la comunicación interna se desarrolla en organizaciones que han sido reconocidas por sus buenas prácticas en este campo en España. Se estructura a través de un análisis evolutivo, de 2014 a 2018, de las empresas premiadas por el Observatorio de Comunicación Interna e Identidad Corporativa (OCl) y de las corporaciones presentes en el ranking de mejores empresas para trabajar en España que elabora Great Place to Work (GPTW). Los objetivos secundarios son:

- Estudiar las empresas reconocidas por el ranking Great Place to Work, así como sus características comunes y la importancia que en ellas tiene la comunicación interna.

- Analizar las entidades reconocidas con los premios del Observatorio de Comunicación Interna y sus rasgos coincidentes.

- Conocer las categorías que emplea el Observatorio de Comunicación Interna para establecer sus distinciones y cuál ha sido su evolución temporal.

- Identificar las prácticas de comunicación interna más habituales y aquellas que se perfilan como tendencias futuras en las empresas presentes en el ranking Great Place to Work y en las premiadas por el Observatorio de Comunicación Interna.

\section{Revisión bibliográfica}

La comunicación corporativa es el eje que rige y posibilita la consecución de logros en las instituciones (Apolo et al., 2017). Por ello la gestión estratégica integral de la comunicación es una pieza clave en la competitividad de las organizaciones y la comunicación interna

"se ha convertido en un elemento dinamizador y de soporte de las estrategias de cualquier organización" (Álvarez-Nobell; Lesta, 2011, p. 12),

que ayuda a transmitir la identidad y los valores de la marca. Esto se debe a que la personalidad corporativa se manifiesta en los actos de la empresa y en su estilo comunicativo, así como en la forma en que se relaciona no sólo con los públicos externos sino con los internos, concretados en sus empleados (Costa, 2018).

La comunicación interna posibilita la adaptación al cambio (Elías; Mascaray, 1998) ya que

"se trata de un elemento clave que sustenta y articula el resto de las estrategias de comunicación de la compañía, cohesionando su estructura interna y ayudando a objetivos concretos" (Peña-Acuña; Caldevilla-Domínguez; Batalla-Navarro, 2017, p. 317).

Abundan los autores que mantienen que la comunicación interna tiene un papel determinante en el sentimiento de pertenencia de los empleados hacia la organización (Ruiz-De-Alba-Robledo, 2013; Karanges et al., 2015; Peña-Acuña; Batalla-Navarro, 2016; Wills-Espinosa et al., 2017), ya que a través de ella la empresa logra su aceptación e integración (Capriotti, 1999) y el aumento del compromiso organizacional (Welch; Jackson, 2007; Neto; Cruz, 2017). Esto se debe a que

"la comunicación interna es un elemento imprescindible para lograr la implicación del personal" (Villafañe, 2000, p. 240). 
En la actualidad este objetivo pasa por fomentar el diálogo y la conversación y por hacer partícipes a los empleados de los diferentes procesos (Quirke, 2017).

La comunicación interna se emplea para cimentar la reputación y crear experiencia de marca. Algunas tendencias en herramientas de comunicación interna son la implementación de intranet social, gamificación, redes sociales para impulsar el engagement y embajadores de marca (Pintado-Blanco; Sánchez-Herrera, 2017), así como realidad virtual o videoconferencia (Castillo-Esparcia, 2008), entre muchas otras. Estas nuevas herramientas vienen a sumarse a otras ya tradicionales (Xifra, 2011) como revistas internas o tablones de anuncios.

Es necesario resaltar que la comunicación interna debe gestionarse desde el departamento de comunicación y no desde el de recursos humanos, como ocurre en algunos casos, aunque la colaboración y el trabajo coordinado entre ambos resulte enriquecedor (Almansa-Martínez, 2005) y los medios de comunicación interna puedan ponerse a disposición de la gestión de trabajadores.

Por otra parte, el concepto de felicidad aplicado al ámbito profesional es complejo y ambiguo, ya que se vincula a aspectos laborales, ambientales y personales (Warr, 2013). A esto se debe que el comportamiento organizativo positivo asociado al rendimiento laboral haya sido abordado desde diversos enfoques, como el de la psicología positiva, que en ocasiones asocian la felicidad al placer y otras veces lo hacen al concepto de bienestar, implicando placer, compromiso y significado (Moccia, 2016). La falta de preocupación en algunas empresas hacia el sufrimiento de los empleados por parte de los responsables de personas y de su abordaje en los procesos de comunicación interna (Gismera-Tierno; Fernández-Fernández; Labrador-Fernández, 2017) es un asunto que merece profundización.

El papel de la comunicación es fundamental en aspectos como la implantación de la responsabilidad social corporativa (RSC), ya que contribuye a la integración de los componentes de la empresa a partir de los mensajes emitidos por la alta dirección (Aceituno-Aceituno et al., 2013) o en la gestión de la calidad total en la empresa, donde los flujos de comunicación interna son básicos para gestionar los nuevos procesos (Nigro-Cardia, 2004). Sin embargo, la importancia en cuanto a la aportación que la comunicación interna puede suponer al bienestar, compromiso y felicidad en términos generales de los trabajadores es un campo amplio en el que investigar, ya que felicidad y trabajo son conceptos que están íntimamente ligados entre sí y vinculados a la realización personal (Fidelis; Borelli; Rech, 2015).

Un modo de observar la evolución y desarrollo de la comunicación interna es recurrir a organismos que reconocen iniciativas en este campo, como el Observatorio de Comunicación Interna, o a empresas de auditoría como Great Place to Work.

\section{Metodología}

Esta investigación se configura como un estudio descriptivo que se apoya en la combinación de métodos de análisis mixtos (Campos-Arenas, 2009) para que, a través de la triangulación de diversas fuentes de datos, sea posible "un conocimiento completo de los fenómenos que se estudian" (Berganza-Conde; Ruiz-San-Román, 2005, p. 33).

El diseño metodológico se basa en el análisis longitudinal (Wimmer; Dominick, 1996) de dos elementos que se toman como marco de referencia para el quinquenio 2014-2018 en cuanto al estudio de la comunicación interna llevada a cabo por las empresas españolas:

- el ranking Great Place to Work, España;

- los premios del Observatorio de Comunicación Interna e Identidad Corporativa de España.

Estas dos entidades son relevantes en el campo de la comunicación interna debido a la amplia trayectoria que las respalda:

- cuentan con una dilatada experiencia, ya que el Observatorio de Comunicación Interna se fundó en 2001 y la versión española del ranking Great Place to Work se edita desde 2002;

- la envergadura de los estudios que publican los convierten en referentes en la esfera profesional de la comunicación interna organizacional en España y también son empleados en el entorno académico (Jaén-Díaz et al., 2006; Salanova, 2009; Ramilo-Méndez, 2012; Medina-Aguerrebere, 2012).

Se han estudiado los instrumentos empleados por las diferentes organizaciones, sus prácticas más habituales, la presencia por sectores en las clasificaciones de ambas entidades y su relación con los entornos de trabajo. Las técnicas de recolección de datos que se han empleado han sido:

- Revisión bibliográfica documental: tanto para establecer el marco teórico de la investigación como para determinar los canales más importantes en comunicación interna.

- Análisis cuantitativo de las empresas recogidas en el ranking y reconocidas por el OCl: la muestra consta de las 250 empresas presentes entre 2014 y 2018 en el ranking y de las 38 galardonadas con los premios del OCI. La distribución anual es la que sigue:

2014: 50 empresas del ranking y 7 premiados

2015: 50 y 8

2016: 50 y 9

2017: 50 y 7

2018: 50 y 7 
Tanto el ranking como los premios se publican en las revistas corporativas de estas entidades, por lo que se han analizado las 10 revistas que abarca el período.

- Entrevistas semiestructuradas a representantes de ambas instituciones: Álvaro Martínez Ortega (Manager del Área de Estudios de GPTW) y Ana Domínguez Castaño (Departamento de Comunicación del Observatorio de Comunicación Interna).

- Análisis cualitativo y cuantitativo de documentación interna de las entidades.

En el estudio del ranking se valoran elementos (tabla 1) como el año, el tamaño de la plantilla o el sector de la empresa.

El ranking GPTW se elabora en España desde 2002 y en Estados Unidos desde 1992 y tiene presencia en 60 países. GPTW desarrolla una certificación derivada de una auditoría donde se valoran requisitos que, según el método propio de la consultora, debe cumplir una empresa para ser considerada un buen lugar para trabajar. Este sistema, que toma la confianza como un indicador de negocio, se configura en dos ejes:

- Trust index: supone 2/3 del peso en la valoración final de cada empresa. Se calcula mediante un cuestionario propuesto al total de la plantilla de la entidad, obteniendo un número de respuestas con un nivel de confianza del $95 \%$ y un 5\% de margen de error. Se basa en cinco pilares: credibilidad, respeto, imparcialidad, orgullo y camaradería, sobre los que incluyen al menos 60 preguntas.

- Culture audit: supone 1/3 de la puntuación global. Recoge la visión de la organización, permitiendo identificar acciones concretas para la evolución de la cultura corporativa.

Para que una entidad pueda ser certificada y optar al ranking debe obtener en el Trust index al menos un $70 \%$ de respuestas positivas (valoradas con 4 ó 5 sobre 5 ) y estar por encima del percentil 50 en la calificación de la evaluación de todas las empresas. De las aproximadamente 70 empresas certificadas cada año en España de unas 300-400 que lo solicitan, las 50 que obtienen mayor puntuación pasan a formar parte del ranking, independientemente de su número de empleados, por lo que la presencia de entidades según su plantilla es variable.

Para estudiar los premios del $\mathrm{OCl}$ se establecen categorías (tabla 2) como la edición y el premio recibido, la empresa premiada, el sector al que pertenece o la práctica que lleva a cabo.
Tabla 1. Tabla de análisis del ranking GPTW

\begin{tabular}{|l|l|}
\hline Año & $2014 / 2015 / 2016 / 2017 / 2018$ \\
\hline Nombre de la empresa & \\
\hline \multirow{5}{*}{ Número de empleados } & $50-100$ \\
& $100-250$ \\
& $250-500$ \\
& $500-1000$ \\
& $>1000$ \\
\hline & Farmacéutico-sanitario \\
& Tecnología \\
& Seguros \\
& Banca/sector financiero \\
& Industria/industria química/reciclaje \\
& Alimentación \\
& Hostelería y restauración \\
& Servicios profesionales/marketing \\
& Recursos humanos \\
& Servicios profesionales/gestión \\
& Gran consumo/electrónica \\
& Retail \\
& Automoción \\
& Telecomunicaciones \\
& Tabaco \\
& Retail/textil \\
& Energético \\
& Transporte \\
& Sistemas de seguridad \\
& Inmobiliaria \\
\hline \multirow{5}{*}{ Sector de la empresa } & \\
\hline
\end{tabular}

Tabla 2. Tabla de análisis de los premios del $O C I$

\begin{tabular}{|c|c|}
\hline Edición - Año & $2014 / 2015 / 2016 / 2017 / 2018$ \\
\hline Categoría & $\begin{array}{l}\text { Campaña de comunicación interna } \\
\text { Estrategia de comunicación interna para la gestión del cambio } \\
\text { Estrategia global de social media y entornos digitales } \\
\text { Estrategia y plataforma online } \\
\text { Herramienta } 2.0 \text { y de social media } \\
\text { Mejor practica de comunicación interna en responsabilidad social empresarial } \\
\text { Mejor publicación en soporte convencional para la comunicación interna } \\
\text { Plan estratégico de comunicación personal "En cascada" } \\
\text { Plataforma y soporte online para la gestión eficaz de la comunicación interna } \\
\text { Política de comunicación interna en pymes } \\
\text { Práctica de comunicación interna en Latinoamérica }\end{array}$ \\
\hline \multicolumn{2}{|l|}{ Premiado } \\
\hline Sector de la empresa & $\begin{array}{l}\text { Banca/servicios financieros } \\
\text { Industria/reciclaje } \\
\text { Retail } \\
\text { Seguros } \\
\text { Telecomunicaciones } \\
\text { Aerolíneas } \\
\text { Alimentación } \\
\text { Construcción } \\
\text { Energético } \\
\text { Farmacéutico-sanitario } \\
\text { Cosmética } \\
\text { Defensa estatal } \\
\text { Gran consumo/electrónica } \\
\text { Hostelería y restauración } \\
\text { Servicios } \\
\text { Servicios profesionales/gestión } \\
\text { Recursos humanos } \\
\text { Transporte }\end{array}$ \\
\hline Práctica & Descripción y herramientas de comunicación empleadas \\
\hline
\end{tabular}


Los premios del $\mathrm{OCl}$ son un reconocimiento a empresas que destacan en su comunicación interna con respecto a diferentes categorías. Comenzaron en 2009 y reciben unas 70-80 candidaturas anuales. Las empresas se postulan y el jurado, compuesto por profesionales de reconocido prestigio, selecciona tres empresas finalistas en cada categoría, entre las que otorga los premios.

En el análisis documental del material obtenido tanto de GPTW como del $\mathrm{OCl}$ (tabla 3 ) se han tenido en cuenta elementos como informes de Trust index, informes de las mejores prácticas, presentaciones internas para clientes de GPTW o informes de los premios del $\mathrm{OCl}$, además del contenido de sus foros.

Tabla 3. Análisis de documentación propia de GPTW y OCI

\begin{tabular}{|l|l|}
\hline \multicolumn{1}{|c|}{ Entidad } & \multicolumn{1}{c|}{ Documentación analizada } \\
\hline Great Place to Work, España & $\begin{array}{l}\text { Informe Trust index 2014-2018 } \\
\text { Informe prácticas de los Best workplaces 2014-2018 } \\
\text { Presentación interna para clientes GPTW } \\
\text { Dossieres de prensa Best workplaces 2014-2018 }\end{array}$ \\
\hline Observatorio de la Comunicación Interna e Identidad Corporativa & $\begin{array}{l}\text { Informes de los premios del OCI 2014-2018 } \\
\text { Foros del OCI 2014-2018 donde se presentan los casos ganadores }\end{array}$ \\
\hline
\end{tabular}

\section{Resultados}

\subsection{Análisis del ranking Great Place to Work 2014-2018}

El ranking GPTW, en sus ediciones de 2014 a 2018, abarca 250 empresas, 50 por año; de ellas el 58,4\%, es decir 146 corporaciones, repiten presencia en el ranking, por lo que a lo largo de los cinco años hay 104 empresas únicas. En el quinquenio estudiado la distribución media de empresas según el volumen de su plantilla es:

- 25\% entre $250-500$ trabajadores

- $24 \%$ entre $100-250$

- $18 \%$ más de 1.000

- $17 \%$ entre $100-250$

$-16 \%$ entre $50-100$

Las empresas que repiten presencia en la clasificación han permanecido en el mismo tramo de plantilla a excepción de AbbVie, que en 2014 tenía de 250 a 500 trabajadores y entre 2015 y 2018 aumentó su plantilla y se sitúa en el rango entre 500 y 1000

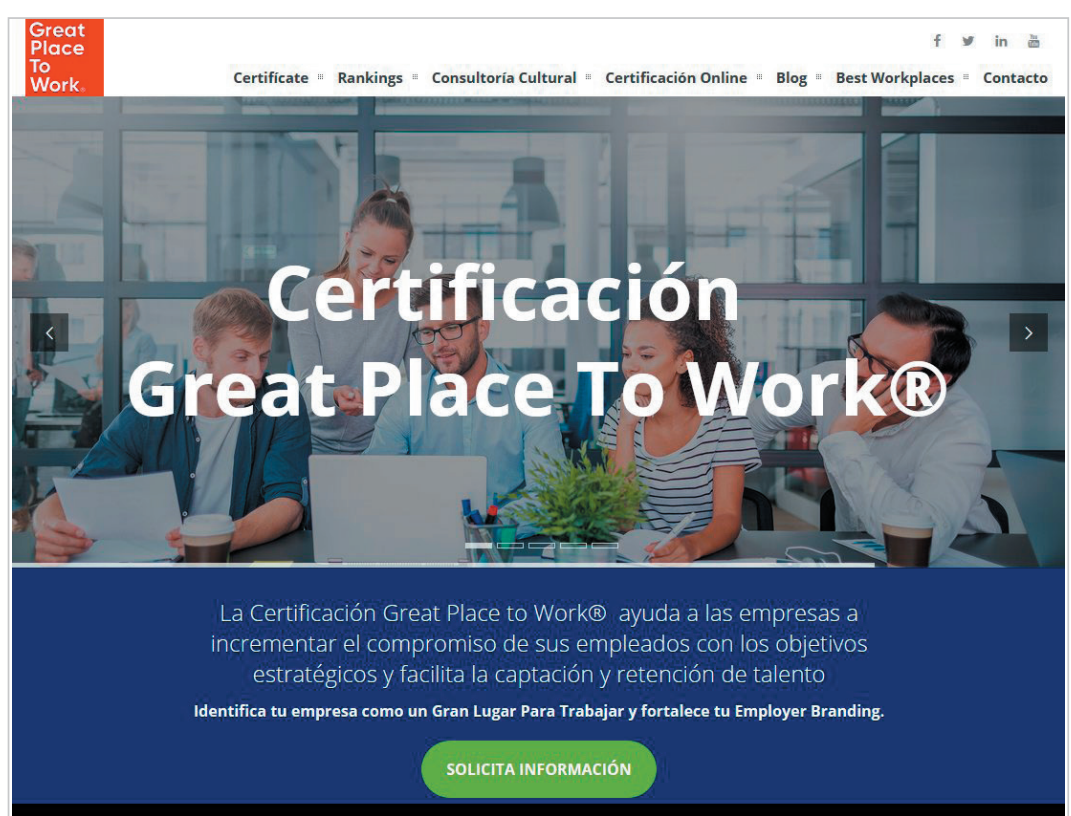

https://greatplacetowork.es empleados.

El análisis revela las mejores prácticas (tabla 4) que de forma global llevan a cabo las empresas que han obtenido un puesto en el ranking durante las últimas cinco ediciones. Estas iniciativas enfocadas a lograr los objetivos de la organización se clasifican en nueve apartados: Hablar, Escuchar, Agradecer, Inspirar, Desarrollar, Cuidar, Celebrar, Compartir y Contratar. En todas ellas están presentes diferentes estrategias, tácticas o herramientas de comunicación interna. Ello indica que en las mejores empresas para trabajar, la comunicación hacia los públicos internos es una constante que impregna las mejores prácticas con el fin de obtener el reconocimiento de los empleados.

Tabla 4. Mejores prácticas de las empresas del ranking GPTW 2014-2018

\begin{tabular}{|l|}
\hline Hablar \\
\hline Comunicación formal (conferencias, convenciones) \\
\hline Flujos de información intraequipos, líderes de opinión de cada área o managers \\
\hline Información corporativa inmediata y trasparente sobre cambios o decisiones \\
\hline Escuchar \\
\hline Reclamar, canales para denuncias internas garantizando anonimato \\
\hline Reuniones periódicas de feedback \\
\hline Instalaciones que fomenten el diálogo (open space, chill out, salas de reuniones informales) \\
\hline
\end{tabular}




\begin{tabular}{|c|}
\hline Agradecer \\
\hline Agradecimiento informal de los managers \\
\hline Dotar de medios de reconocimiento para los equipos \\
\hline Agradecimientos institucionales o premios por méritos \\
\hline Inspirar \\
\hline Iniciativas para reformar la cultura y vincular emocionalmente a los empleados \\
\hline Fomentar la colaboración \\
\hline Hacer partícipes a los empleados de la contribución al negocio y a la sociedad \\
\hline Desarrollar \\
\hline Dirigir a todos los niveles de la organización, segmentando colectivos específicos \\
\hline Formación teórica y práctica en conocimientos, habilidades y comportamientos \\
\hline Involucrar a los empleados en el diseño del plan de desarrollo \\
\hline Cuidar \\
\hline Ayuda en situaciones especiales o difíciles \\
\hline Campañas o iniciativas para promover la salud y bienestar \\
\hline Políticas de flexibilidad y conciliación \\
\hline Adaptar facilities (entorno y nuevas formas de trabajo) \\
\hline Celebrar \\
\hline Momentos únicos y especiales (aniversarios, lanzamientos de productos, logros...) \\
\hline Celebraciones temáticas \\
\hline Celebraciones informales por equipos \\
\hline Compartir \\
\hline Políticas de responsabilidad social corporativa \\
\hline Involucrar a los empleados en las iniciativas \\
\hline Fomentar colaboración con los grupos de interés \\
\hline Contratar \\
\hline Cuidar la experiencia de los candidatos \\
\hline Utilizar medios digitales y datos \\
\hline Utilizar referencias de empleados \\
\hline Involucrar a los empleados en el proceso \\
\hline Realizar una comunicación trasparente del proceso \\
\hline
\end{tabular}

Elaborado a partir de datos de GPTW

La relevancia de estas prácticas a nivel de comunicación interna puede establecerse del siguiente modo: Escuchar, Agradecer, Inspirar, Cuidar, Celebrar, Compartir, Hablar, Desarrollar y Contratar. Álvaro Martínez (comunicación personal, 21 de marzo de 2019) resalta en la entrevista el papel clave de la comunicación interna ya que cuando a los empleados se les proporciona la información adecuada con total transparencia se alinean con el papel que tienen en la organización.

El análisis del cuestionario Trust index de las empresas del ranking indica que el promedio total es de 82 puntos sobre 100 entre 2014 y 2017 y un punto inferior (81) en 2018. A lo largo de los cinco años en todas estas secciones la puntuación ha sido superior a 79, siendo el promedio de Orgullo el que alcanza mejor valoración y el de Imparcialidad el que menos (gráfico 1).

En cuanto a la calificación global de los empleados sobre si consideran las empresas como un lugar 'Excelente' para trabajar el promedio es de 87 puntos sobre 100, aunque ha descendido de 88 puntos en 2014 a 85 en 2018. Otras cuestiones como si 'los superiores son accesibles y es fácil hablar con ellos', si 'cuando te incorporas a la empresa, te sientes bien acogido/a', si se celebran 'los acontecimientos especiales' o si 'es un lugar psicológica y emocionalmente saludable para trabajar' también obtienen respuestas muy positivas a lo largo de los años. En las preguntas sobre si los empleados son tratados de manera justa independientemente de su edad, raza, sexo u orientación sexual las puntuaciones son muy altas, superiores a 90 puntos. Igual ocurre en la cuestión sobre si reciben un buen trato independientemente de la posición que ocupen en la empresa. Las puntuaciones, aunque siempre por encima de 70 puntos, son más bajas en aspectos como si se anima a equilibrar vida personal y laboral, si todos tienen la oportunidad de recibir un reconocimiento especial o si los superiores 'incentivan, consideran y responden sinceramente a nuestras ideas y sugerencias'. 
Según Álvaro Martínez (comunicación personal, 21 de marzo de 2019):

"Ias compañías con un Trust index superior a 80 puntos generan retornos financieros de hasta 3 veces más a sus accionistas que empresas con un Trust index menor".

Las empresas analizadas pertenecen a 21 sectores productivos, en los que destacan el farmacéutico-sanitario y tecnología-seguros, seguidos por otros que van desde banca-sector financiero, a industria, retail, servicios profesionales, tecnología o transportes.

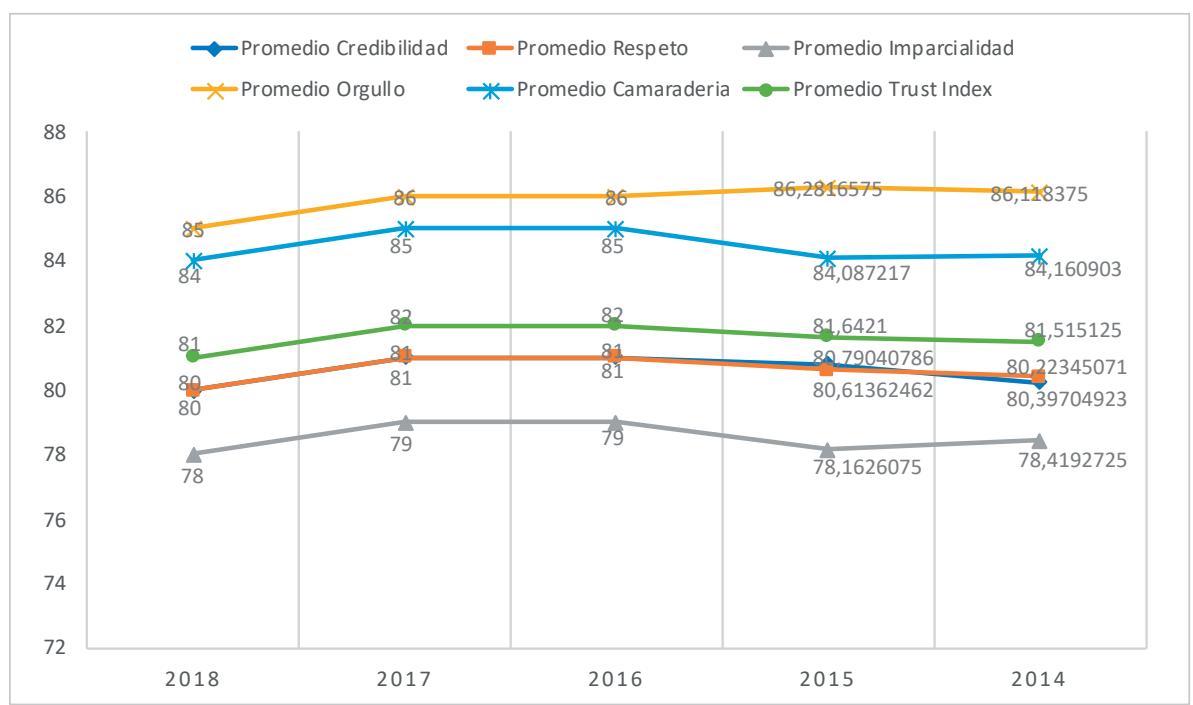

Gráfico 1. Promedios en los apartados del cuestionario Trust index por las empresas presentes en el ranking Great Place to Work entre 2014 y 2018.

Elaborado a partir de datos de GPTW.

La importancia cuantitativa de los sectores productivos (tabla 5) experimenta variaciones si se analiza el tamaño de la plantilla. Hay segmentos como el de las empresas de 50 a 100 trabajadores donde lo preeminente son tecnológicas o el de más de 1.000 empleados, donde las empresas que se dedican a los recursos humanos ocupan el segundo puesto $(19,6 \%)$. Un caso significativo es el del retail, que a nivel global supone únicamente un $3,2 \%$ y en el segmento de plantillas que superan los 1.000 integrantes asciende hasta un $17,4 \%$.

En cifras totales destaca el sector farmacéutico en todos los casos y el sector tecnología en las empresas de hasta 500 empleados. Los demás sectores tienen grandes variaciones debidas al tamaño de las plantillas, incluso con ausencias en todos los rangos de gran parte de ellos, ya que hay una media de 9,8 sectores que no aparecen en cada segmento.

Tabla 5. Sectores presentes en el ranking Great Place to Work 2014-2018

\begin{tabular}{|c|c|c|c|c|c|c|}
\hline \multirow{2}{*}{ Sectores } & \multirow{2}{*}{$\begin{array}{c}\% \text { presencia } \\
\text { global }\end{array}$} & \multicolumn{5}{|c|}{ \% presencia según plantilla } \\
\hline & & $50-100$ & $100-250$ & $250-500$ & $500-1000$ & $>1000$ \\
\hline Farmacéutico-sanitario & 24,8 & 12,2 & 25,0 & 27,4 & 35,7 & 21,8 \\
\hline Tecnología & 14,4 & 29,3 & 18,3 & 17,7 & 4,8 & 0 \\
\hline Seguros & 10,8 & 0 & 10,0 & 9,7 & 21,4 & 13,0 \\
\hline Banca/sector financiero & 5,2 & 4,9 & 5,0 & 6,5 & 2,4 & 6,5 \\
\hline Industria/industria química/reciclaje & 5,2 & 0 & 18,3 & 0 & 7,1 & 0 \\
\hline Alimentación & 5,2 & 2,4 & 5,0 & 6,5 & 9,5 & 2,2 \\
\hline Hostelería-restauración & 4,4 & 9,8 & 10,0 & 1,6 & 0 & 0 \\
\hline Servicios profesionales/marketing & 4,4 & 9,8 & 1,7 & 9,7 & 0 & 0 \\
\hline Recursos humanos & 4,0 & 0 & 1,7 & 0 & 0 & 19,6 \\
\hline Servicios profesionales/gestión & 3,2 & 12,2 & 1,7 & 0 & 0 & 4,3 \\
\hline Gran consumo/electrónica & 3,2 & 2,4 & 1,7 & 4,8 & 7,1 & 0 \\
\hline Retail & 3,2 & 0 & 0 & 0 & 0 & 17,4 \\
\hline Automoción & 2,8 & 9,8 & 0 & 4,8 & 0 & 0 \\
\hline Telecomunicaciones & 2,0 & 0 & 1,7 & 0 & 2,4 & 6,5 \\
\hline Tabaco & 1,6 & 0 & 0 & 3,2 & 4,8 & 0 \\
\hline Retail/textil & 1,6 & 0 & 0 & 0 & 0 & 8,7 \\
\hline Energético & 1,6 & 0 & 0 & 6,5 & 0 & 0 \\
\hline Transporte & 1,2 & 2,4 & 0 & 1,6 & 2,4 & 0 \\
\hline Sistemas de seguridad & 0,4 & 2,4 & 0 & 0 & 0 & 0 \\
\hline Inmobiliaria & 0,4 & 0 & 0 & 0 & 2,4 & 0 \\
\hline Cosmética & 0,4 & 2,4 & 0 & 0 & 0 & 0 \\
\hline
\end{tabular}

Elaborado a partir de datos de GPTW 
Otro resultado relevante es la permanencia de las empresas en el ranking, cuya media es de 2,4 años y varía según el número de empleados (gráficos 2 y 3 ).

Los datos más nivelados respecto al tamaño de la plantilla se encuentran en las empresas que tienen presencia un año en el ranking, ya que no hay grandes disparidades. A medida que aumenta la permanencia se incrementa la diferencia ya que las empresas que aparecen dos años son mayoritariamente grandes (más de 500 trabajadores). En lo que se refiere a tres años de permanencia la presencia vuelve a estar equilibrada y en cuatro y cinco años los resultados se polarizan.

\subsection{Análisis de los premios del Obser- vatorio de Comunicación Interna 2014- 2018}

En los premios del OCl de 2014 a 2018 ha habido 38 galardones y 37 empresas premiadas, ya que ING Direct recibió dos premios en 2014 (Campaña de comunicación interna y Herramienta 2.0 y de social media).

Los principales proyectos premiados por el $\mathrm{OCl}$ (gráfico 4) se desglosan en:

- campañas globales de comunicación interna con diversos elementos y soportes $(19,4 \%)$;

- mejoras generales de las herramientas internas $(13,9 \%)$;

- revistas internas online o físicas $(11,1 \%)$;

- implementación o mejora de la intranet;

- acciones con un concurso como eje;

- aplicaciones móviles para empleados, red social interna y embajadores de marca.

Entre las acciones que tienen un único ejemplo $(2,8 \%)$ destacan cuatro campañas sobre mejoras del clima laboral o la plantilla:

- de reconocimiento de empleados;

- de racionalización de horarios y fomento de la conciliación;

- de promoción de la salud y de prevención de riesgos laborales;

- de promoción de la seguridad vial y aumento de pertenencia entre los trabajadores.

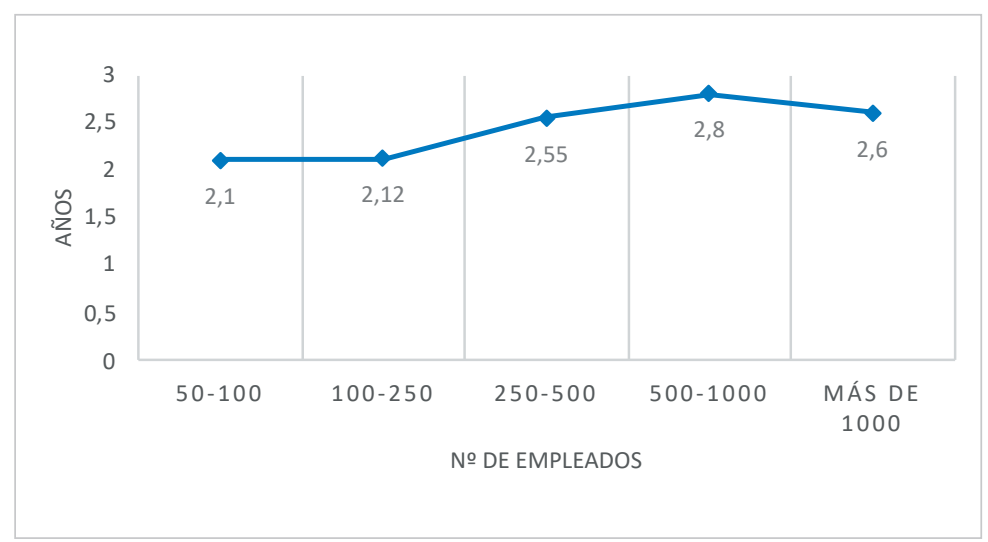

Gráfico 2. Permanencia en el ranking Great Place to Work entre 2014 y 2018. Elaborado a partir de datos de GPTW.

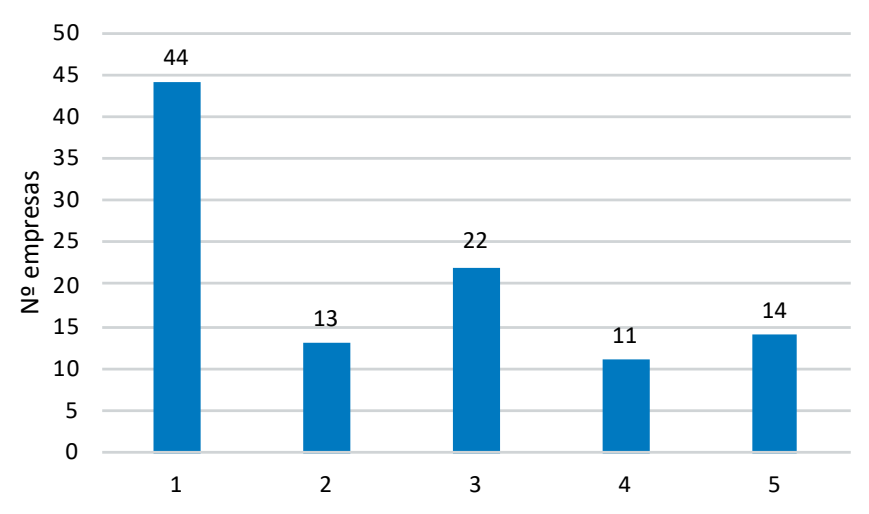

№ años con presencia en el ranking Great Place to Work

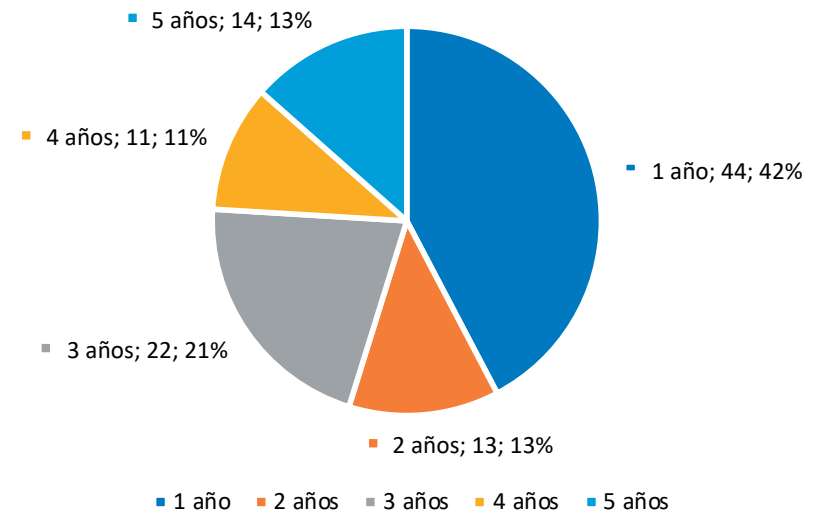

Gráfico 3. Permanencia de las empresas en el ranking GPTW entre 2014 y 2018. Elaborado a partir de datos de GPTW.

Así, un $11,1 \%$ de los premios reconocen acciones para mejorar la experiencia de los empleados.

En los sectores de las empresas premiadas destaca banca (23,7\%), al que pertenecen 9 organizaciones. El resto apenas se repiten, ya que lo hacen en tres ocasiones $(7,9 \%)$ industria, retail y seguros; en dos (5,3\%) farmacéutico-sanitario, telecomunicaciones, aerolíneas, construcción, alimentación y energético; y en una (2,6\%) gran consumo, hostelería, servicios profesionales, recursos humanos, transporte, cosmética, defensa estatal y servicios.

Al analizar los sectores en base a los premios, el más numeroso es banca $(63,6 \%$ de las categorías) seguido por industria/reciclaje (27,3\%). Hay 9 sectores que sólo aparecen en una categoría (9\%) y 7 sectores que aparecen en 2 categorías.
Las empresas con una presencia más estable a lo largo del tiempo en el ranking GPTW son las que superan los 500 trabajadores 


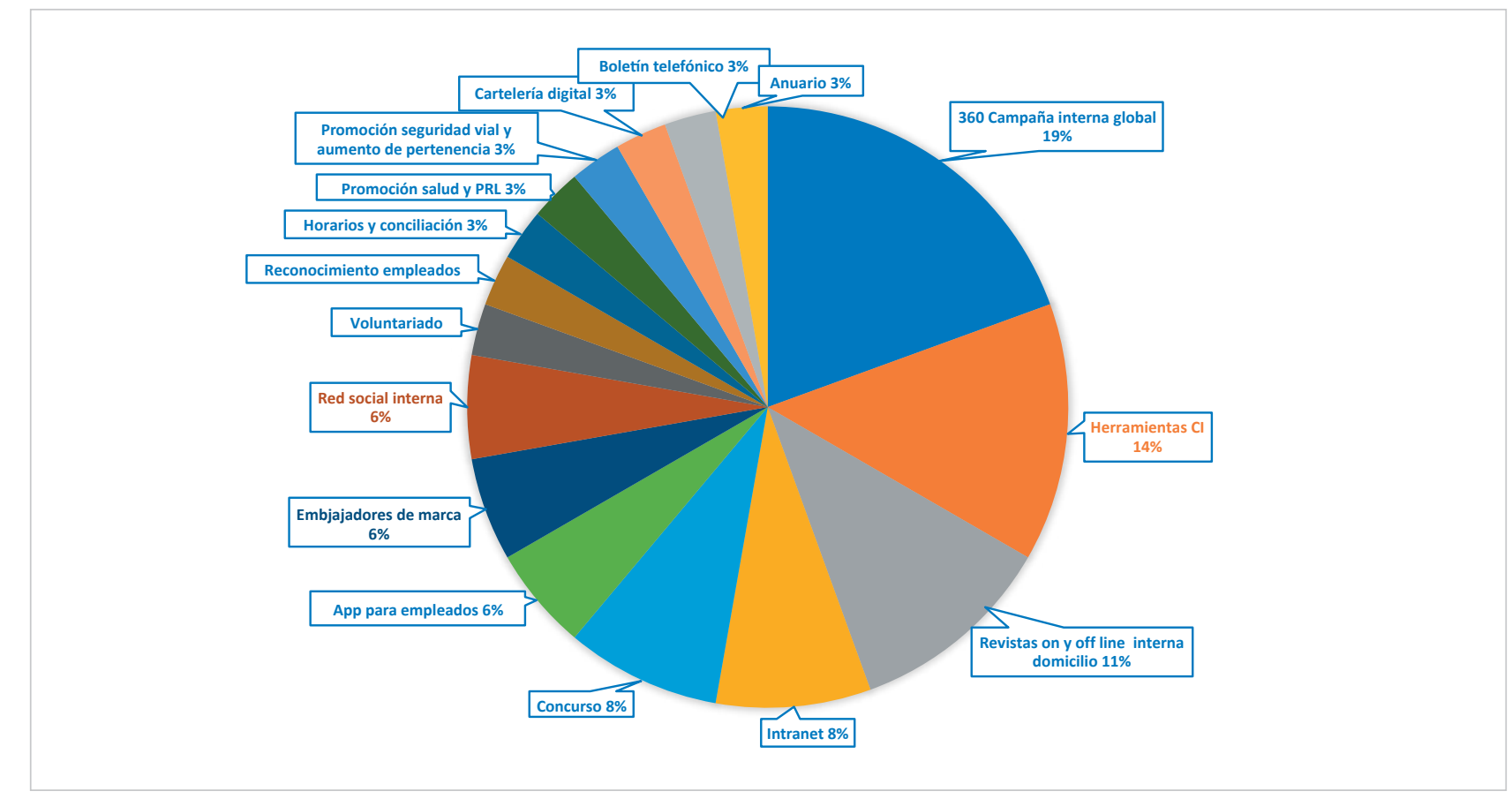

Gráfico 4. Acciones premiadas por el Observatorio de Comunicación Interna 2014-2018.

Elaborado a partir de datos del $O C I$.

Los 11 tipos de premio han variado a lo largo de las ediciones:

- Campaña de comunicación interna (2014-2018);

- Estrategia de comunicación interna para la gestión del cambio (2014-2018);

- Mejor práctica de comunicación interna en responsabilidad social empresarial (2014-2018);

- Mejor publicación física para la comunicación interna (2014-2018);

- Práctica de comunicación interna en Latinoamérica (2015-2018);

- Política de comunicación interna en pymes (2017-2018);

- Estrategia y plataforma online (2017-2018);

- Estrategia global de social media y entornos digitales (2014-2016);

- Plataforma y soporte online para la gestión eficaz de la comunicación interna (2014-2016);

- Herramienta 2.0 y de social media (2014) y

- Plan estratégico de comunicación personal 'En cascada' (2015).

En el histórico de categorías, un 36,6\% (4) son online y un $63,6 \%$ no lo son, siendo una de ellas expresamente convencional, aunque también categorías que no son online premian campañas que incluyen medios digitales complementarios. Se percibe una evolución en los galardones:

- a partir de 2017 se unifican los referentes a acciones online, por lo que se elimina la sobredimensión que recibían en algunas ediciones y se equipara su importancia a otras herramientas;

- se amplia el alcance incluyendo las categorías de Latinoamérica y pymes.

4.3. Análisis comparativo del ranking Great Place to Work y de los premios del Observatorio de Comunicación Interna 2014-2018

Entre el ranking GPTW y el $\mathrm{OCl}$ tan sólo se repiten 4 empresas: $D K V$ Seguros, ING Bank, LG Electronics y Voda-
Los principales proyectos premiados por el $\mathrm{OCl}$ son campañas globales de comunicación interna con diversos elementos y soportes

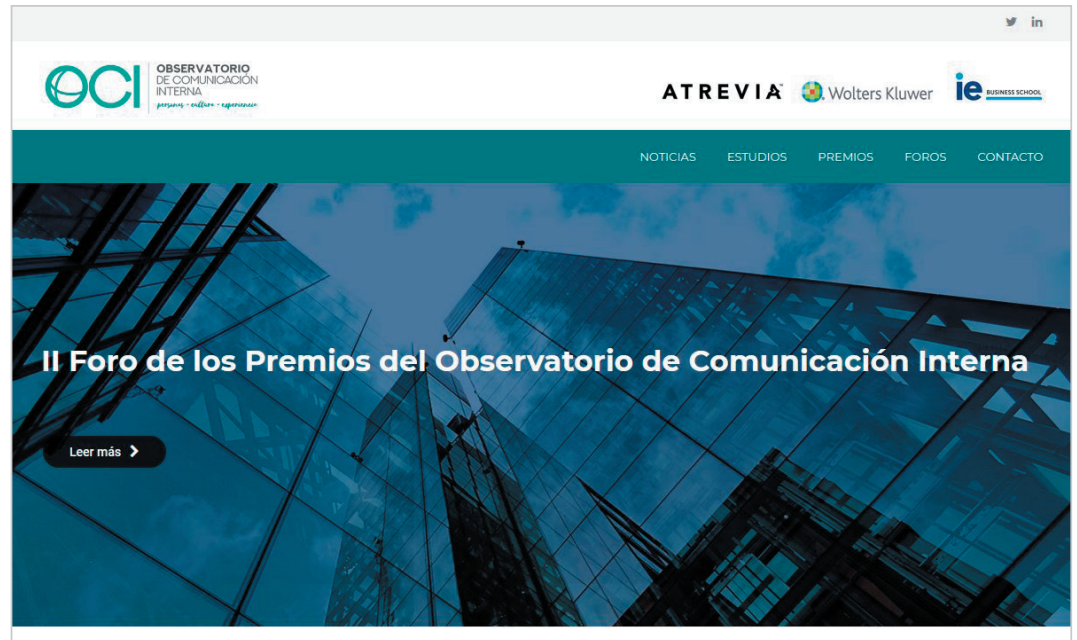

http://www.observatoriocomunicacioninterna.es 
fone. En cuanto a las prácticas e instrumentos de comunicación interna existe una amplia coincidencia en los resultados obtenidos del análisis comparativo (tabla 6).

Tabla 6. Herramientas de comunicación interna más empleadas

\begin{tabular}{|l|l|}
\hline \multicolumn{1}{|c|}{ Great Place to Work } & \multicolumn{1}{c|}{ OCI } \\
\hline Habilidades de comunicación y escucha activa & $\begin{array}{l}\text { Campañas internas globales para cambiar actitudes y propiciar el } \\
\text { Líderes de opinión y embajadores de marca }\end{array}$ \\
Redes sociales internas & Revistas internas \\
Intranet & Embajadores de marca \\
Revistas internas & Intranet y blogs corporativos \\
Canales de TV & Redes sociales y aplicaciones internas \\
Empleo de pantallas, cartelería o el fondo de pantalla de los ordena- & Eventos y concursos para empleados \\
dores de los propios empleados & Cartelería digital y otras herramientas novedosas de Cl, como panta- \\
Campañas de merchandising & llas y presentaciones digitales interactivas \\
Eventos & Formación online \\
Oficinas que fomentan la cooperación, co-creación y colaboración & Merchandising \\
& Canal de TV \\
\hline
\end{tabular}

Elaborado a partir de datos de GPTW y OCI

Las tendencias en comunicación interna (A. Martínez, comunicación personal, 21 de marzo de 2019) se orientan a analizar la experiencia de empleado para aumentar su confianza e identificación a través de tecnología y aplicaciones cada vez más interactivas y ágiles, que permitan participar, hablar, escuchar y trabajar en remoto, como las redes sociales o el portal del empleado. Otros puntos destacados son las instalaciones y espacios inspiradores que fomenten la comunicación, colaboración e innovación, y el trabajo con los líderes para aumentar la cercanía, trasparencia y métodos de trabajo más ágiles.

La presencia de los sectores productivos de las empresas estudiadas en ambas categorías es muy desigual (gráfico 5), ya que la importancia de algunos es muy dispar y un $44 \%$ no está presente en alguna de las dos secciones. Las mayores diferencias se dan en los sectores con más peso tanto en una como en otra categoría: en GPTW el más importante es el farmacéutico-sanitario (24,8\%), que sin embargo supone un 5,3\% en el OCl; entre los premiados por el $\mathrm{OCl}$ destaca el sector banca $(23,7 \%)$, que en el ranking apenas supone un $5,2 \%$. Otros sectores muy dispares son tecnología, servicios profesionales/marketing, aerolíneas y construcción.
La presencia de los sectores productivos es muy desigual: en GPTW el más importante es el farmacéutico-sanitario y en los premios del $\mathrm{OCl}$ la banca

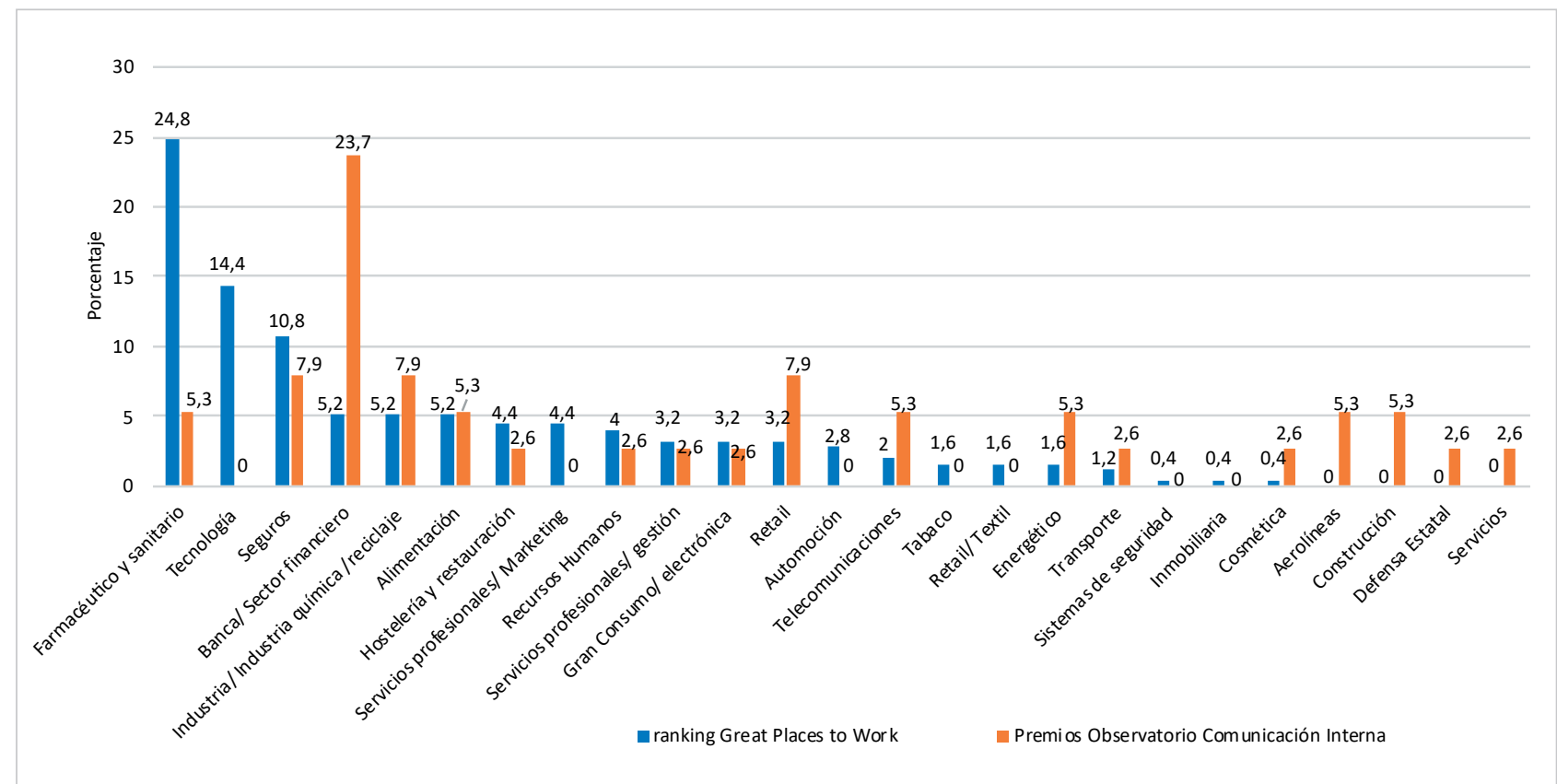

Gráfico 5. Comparativa de la presencia por sectores en el ranking Great Place to Work y en los premios del Observatorio de Comunicación Interna 2014-2018.

Elaborado a partir de datos de GPTW y OCI. 


\section{Conclusiones y discusión}

Este trabajo incide en que la implementación de estrategias de comunicación interna, tal y como señalan otras investigaciones (Capriotti, 1999; Villafañe, 2000; Welch; Jackson, 2007; Ruiz-De-Alba-Robledo, 2013; Karanges et al., 2015; Peña-Acuña; Batalla-Navarro, 2016; Wills-Espinosa et al., 2017; Neto; Cruz, 2017), contribuye a generar compromiso e implicación en los trabajadores y se configura como un elemento fundamental en la transformación empresarial, coincidiendo con el posicionamiento de trabajos previos sobre este tema (Álvarez-Nobell; Lesta, 2011; Apolo et al., 2017).

En respuesta a los objetivos señalados, esta investigación revela una coincidencia en las herramientas y prácticas empleadas en la comunicación interna de las organizaciones estudiadas. También destaca la importancia que otorgan a la gestión integral del cambio a través de campañas globales dirigidas a sus empleados y mandos con el objetivo de desarrollar tanto la escucha activa como la transformación del clima corporativo. Estos resultados son acordes a lo planteado por Elías y Mascaray (1998) en cuanto al papel de la comunicación interna para posibilitar la adaptación al cambio de las organizaciones.

Según este estudio, el uso de aplicaciones digitales está equilibrado con el empleo de medios físicos convencionales, aunque suelen aplicarse de forma combinada en estrategias integrales. Estos resultados perfilan como las más destacadas los embajadores de marca, las redes sociales internas, las aplicaciones móviles internas, el uso de intranet, los eventos, el uso de pantallas y cartelería digital y las revistas internas. Además, indican las tendencias futuras en cuanto a sistemas de comunicación interna, que se concretan en el uso de nuevas tecnologías interactivas, el diseño de espacios e instalaciones que fomenten los flujos comunicativos y el trabajo con los líderes para aumentar la transparencia y la agilidad.

Las líneas generales que seguirá la comunicación interna, según los resultados obtenidos, se centrarán en fomentar la comunicación y la experiencia del empleado para obtener su confianza y que sus intereses se alineen con los de la compañía. Esto concuerda con lo planteado por Quirke (2017) respecto a la necesidad de hacer partícipes a los trabajadores sobre los procesos de la marca.

A tenor de los resultados se concluye que el ranking Great Place to Work es más relevante en empresas de los sectores farmacéutico-sanitario, tecnología y seguros; por su parte, los premios del Observatorio de Comunicación Interna, pese al predominio del sector bancario, están mucho más diversificados. Así, no existe una preferencia por obtener este reconocimiento por parte de empresas de sectores concretos, mientras que la certificación de GPTW parece ser de relevancia en determinados mercados por la participación que genera.

De cara a futuras investigaciones se sugiere profundizar en la relación entre comunicación interna y felicidad de los empleados en diferentes sectores y contextos. También se pueden incluir otras variables como la percepción de la felicidad y el bienestar por parte de los trabajadores.

\section{Referencias bibliográficas}

Aceituno-Aceituno, Pedro; Cea-Moure, Ramiro; Casado-Sánchez, José-Luis; Ruiz-de-Azcárate-Varela, Carmen (2013). "La comunicación como factor clave en la implantación de la responsabilidad social corporativa: el caso de Crédit Agricole España". El profesional de la información, v. 22, n. 4, pp. 326-332.

https://doi.org/10.3145/epi.2013.jul.08

Almansa-Martínez, Ana (2005). "Relaciones públicas y gabinetes de comunicación". Anàlisi: quaderns de comunicació $i$ cultura, v. 32, pp. 117-132.

https://www.raco.cat/index.php/analisi/article/viewFile/15175/179895

Álvarez-Nobell, Alejandro; Lesta, Laura (2011). “Medición de los aportes de la gestión estratégica de comunicación interna a los objetivos de la organización". Palabra clave, v. 14, n. 1, pp. 11-30.

https://www.redalyc.org/articulo.oa?id=64920732002

Apolo, Diego; Báez, Valerie; Pauker, Lizeth; Pasquel, Gabriela (2017). “Gestión de comunicación corporativa: consideraciones para el abordaje de su estudio y práctica". Revista latina de comunicación social, n. 72, pp. 521-539.

https://doi.org/10.4185/RLCS-2017-1177

Berganza-Conde, María-Rosa; Ruiz-San-Román, José A. (2005). Investigar en comunicación: guía práctica de métodos y técnicas de investigación social en comunicación. Madrid: McGraw Hill. ISBN: 8448198255 
Campos-Arenas, Agustín (2009). Métodos mixtos de investigación: integración de la investigación cuantitativa y la investigación cualitativa. Magisterio Editorial. ISBN: 9789582005795

Capriotti, Paul (1999). Planificación estratégica de la imagen corporativa. Ariel Comunicación. ISBN: 8434412756 http://www.bidireccional.net/Blog/PEIC_4ed.pdf

Castillo-Esparcia, Antonio (2008). “Estudio sobre el uso de las tecnologías de la comunicación en la comunicación interna". Anagramas. Rumbos y sentidos de la comunicación, v. 6, n. 12, pp. 91-103.

https://dialnet.unirioja.es/servlet/articulo?codigo $=4851611$

Cheney, George; Zorn Jr., Theodore E.; Planalp, Sally; Lair, Daniel J. (2008). "Meaningful work and personal/social weII-being organizational communication engages the meanings of work". Annals of the International Communication Association, v. 32, n. 1, pp. 137-185.

https://doi.org/10.1080/23808985.2008.11679077

Costa, Joan (2018). “Creación de la imagen corporativa. El paradigma del siglo XXI”. Razón y palabra, v. 22, n. 1_100, pp. 356-373.

http://revistarazonypalabra.com/index.php/ryp/article/view/1160

Elías, Joan; Mascaray, José (1998). Más allá de la comunicación interna: diez estrategias para la implantación de valores y la conquista del comportamiento espontáneo de los empleados. Barcelona: Gestión 2000. ISBN: 9788480882545

Fidelis, Andréa-Cristina; Borelli, Verena-Alice; Rech, Jane (2015). "A relação entre felicidade e trabalho: Um estudo exploratório com profissionais ativos e aposentados". En: Mostra de Iniciação Científica, Pós-graduação, Pesquisa e Extensão, n. 4, pp. 1-18.

https://doi.org/10.18226/610001/MOSTRAXV.2015.83

Fisher, Cynthia D. (2010). "Happiness at work". International journal of management reviews, v. 12, n. 4, pp. $384-412$. https://doi.org/10.1111/j.1468-2370.2009.00270.x

Gismera-Tierno, Eduardo; Fernández-Fernández, José-Luis; Labrador-Fernández, Jesús (2017) “Comunicación interna, sufrimiento y gestión de personas. Una aportación desde la filosofía". Revista internacional de investigación en comunicación. aDResearch ESIC, v. 16, n. 16, pp. 116-135.

https://doi.org/10.7263/adresic-016-006

Jaén-Díaz, Marian; Luceño-Moreno, Lourdes; Martín-García, Jesús; Rubio-Valdehita, Susana (2006). “La comunicación interna como herramienta estratégica al servicio de las organizaciones". eduPsykhé: Revista de psicología y psicopedagogía, v. 5, n. 1, pp. 3-32.

https://dialnet.unirioja.es/servlet/articulo?codigo $=2010122$

Karanges, Emma; Johnston, Kim; Beatson, Amanda; Lings, lan (2015). "The influence of internal communication on employee engagement: A pilot study". Public relations review, v. 41, n. 1, pp. 129-131.

https://doi.org/10.1016/j.pubrev.2014.12.003

Malhotra, Neeru; Ackfeldt, Anna-Lenna (2016). "Internal communication and prosocial service behaviors of front-line employees: Investigating mediating mechanisms". Journal of business research, v. 69, n. 10, pp. 4132-4139.

https://doi.org/10.1016/j.jbusres.2016.03.038

Medina-Aguerrebere, Pablo (2012). "El valor estratégico de la comunicación”. Revista de comunicación y salud, v. 2, n. 1, pp. 19-28.

https://dialnet.unirioja.es/servlet/articulo?codigo=3818627

Moccia, Salvatore (2016). “Felicidad en el trabajo". Papeles del psicólogo, v. 37, n. 2, pp. 143-151.

https://www.redalyc.org/html/778/77846055007

Neto, Cátia-Filipa; Cruz, Sofía-Alexandra (2017). “Comunicação interna e comprometimento organizacional: o caso da autoridade para as condições do trabalho". Sociologia, n. 34, pp. 47-72.

https://doi.org/10.21747/08723419/soc34a3

Nigro-Cardia, Adriana (2004). “Comunicação interna e gestão da qualidade total: o caso Volkswagen - unidade Resende". Production, v. 14, n. 2, pp. 6-17.

https://doi.org/10.1590/S0103-65132004000200002

Peña-Acuña, Beatriz; Batalla-Navarro, Pablo (2016). Dirección de comunicación y habilidades directivas. Dykinson. ISBN: 9788490858400

Peña-Acuña, Beatriz; Caldevilla-Domínguez, David; Batalla-Navarro, Pablo (2017). "Tres casos de empresas internacionales con éxito: estudio de las estrategias de comunicación interna". Chasqui: Revista latinoamericana de comunicación, n. 134, pp. 315-330.

https://dialnet.unirioja.es/descarga/articulo/5974552.pdf 
Pintado-Blanco, Teresa; Sánchez-Herrera, Joaquín (2017). Nuevas tendencias en comunicación estratégica. ESIC Editorial. ISBN: 9788417129354

Proctor, Christine (2014). Effective organizational communication affects employee attitude, happiness, and job satisfaction. Doctoral dissertation, Southern Utah University. Department of Communication.

https://cutt.ly/6ei6hEQ

Quirke, Bill (2017). Making the connections: using internal communication to turn strategy into action. Routledge. ISBN: 9780566087806

Ramilo-Méndez, Nicolás (2012). "10 años de Great Place to Work en España”. Capital humano: revista para la integración y desarrollo de los recursos humanos, v. 25, n. 266, pp. 54-59.

Ruiz-De-Alba-Robledo, José-Luis (2013). “El compromiso organizacional: un valor personal y empresarial en el marketing interno". Revista de estudios empresariales, 2a época, n. 1, pp. 67-86.

https://revistaselectronicas.ujaen.es/index.php/REE/article/view/847/808

Salanova, Marisa (2009). “Organizaciones saludables, organizaciones resilientes”. Gestión práctica de riesgos laborales, n. 58, pp. 18-23.

http://repositori.uji.es/xmlui/handle/10234/73232

Salas-Vallina, Andrés; Alegre, Joaquín; Fernández-Guerrero, Rafael (2018). "Happiness at work in knowledge-intensive contexts: Opening the research agenda". European research on management and business economics, v. 24, n. 3, pp. 149-159.

https://doi.org/10.1016/j.iedeen.2018.05.003

Villafañe, Justo (2000). Imagen positiva. Gestión estratégica de la imagen de las empresas. Pirámide. ISBN: 97884368 12503

Warr, Peter (2013) "Fuentes de felicidad e infelicidad en el trabajo: una perspectiva combinada". Revista de psicología del trabajo y de las organizaciones, v. 29, n. 3, pp. 99-106.

https://doi.org/10.5093/tr2013a15

Welch, Mary; Jackson, Paul R. (2007). “Rethinking internal communication: A stakeholder approach”. Corporate communications: An international journal, v. 12, n. 2, pp. 177-198.

https://doi.org/10.1108/13563280710744847

Wills-Espinosa, Nicola; Ceballos-Icaza, María-lleana; Sadi, Gabriel; Ancin-Adell, Irene (2017). "La relación entre la satisfacción con la comunicación interna y el compromiso organizacional: el caso de una universidad ecuatoriana". Austral comunicación, junio, v. 6, n. 1, pp. 133-160.

https://doi.org/10.26422/aucom.2017.0601.wil

Wimmer, Roger D.; Dominick, Joseph R. (1996). La investigación científica de los medios de comunicación: una introducción a sus métodos. Bosch. ISBN: $847676359 \mathrm{X}$

Xifra, Jordi (2011). Manual de relaciones públicas e institucionales. Madrid: Tecnos. ISBN: 9788430972135

http://abrapcorp.org.br/arquivos/manual-rrpp.pdf

\section{Cronología de la Documentación Española}

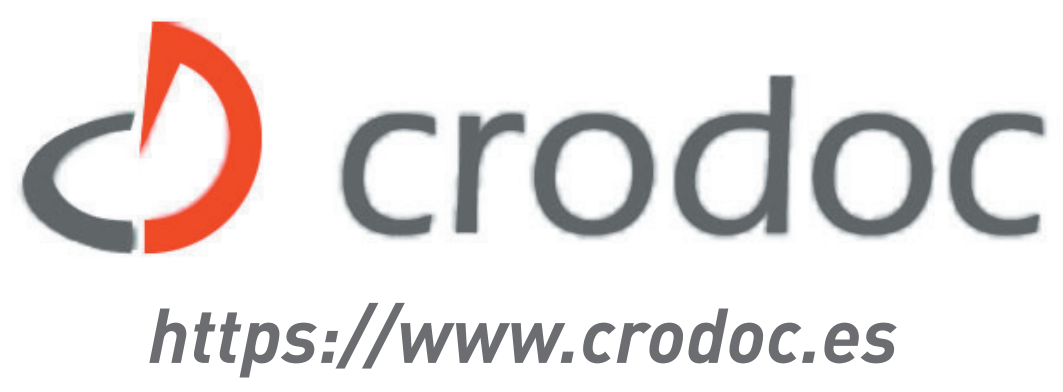

